\title{
Acute Myopericarditis an Uncommon Presentation of Severe Leptospirosis - A Case Report and Literature Review
}

\author{
Cavalcanti S*, L Lerena V and Gomez C \\ Division of Infectious Disease and Tropical Medicine, Centro Médico Naval "CMST", Lima, Perú
}

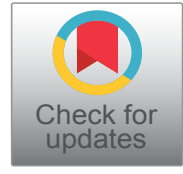

*Corresponding author: Cavalcanti Sofia, MD, Division of Infectious Disease and Tropical Medicine, Centro Médico Naval, Av Venezuela, s/n Bellavista, Lima, Peru

\begin{abstract}
Cardiovascular involvement by Leptospira is infrequent. However the statistics are under estimated. This paper reports a case of a 39 years old male, who works as a Marine and had traveled to a tropical endemic area for Leptospira in Peru. He was initially admitted with fever, headache and myalgias. He was diagnosed of Leptospirosis (Ig M positive and MAT positive) and malaria and receive specific treatment for both diseases (Doxycycline, Cloroquine, Primaquine). Ten days later he was readmitted with severe leptospirosis when he presented mild chest pain with no pulmonary symptoms. Echocardiography showed pericardial fluid (aprox $300 \mathrm{cc}$ ) with no tamponade physiology and pericardial thickening. EKG showed supraventricular arrhythmia (with normal previous EKG) and CPK -M, myoglobin and troponin -I were elevated. There are several reports of cardiovascular involvement in severe leptospirosis but most patients are asymptomatic. Just two cases in 1996 were described, after 20 years we describe a similar case worldwide, and the first time in Peruvian studies. In conclusion we can say that it is very important to follow all patients with uncomplicated leptospirosis and evaluate for cardiac involvement at least with a baseline EKG and cardiac enzymes, to have a comparative study in case they decompensate.
\end{abstract}

\section{Keywords}

Myocarditis and pericarditis, Leptospirosis, EKG, Echocardiography

\section{Background}

Leptospirosis is a potentially fatal disease which can cause multi-organ dysfunction. In 2010, a paper made in Peru, said that the prevalence of Leptospirosis in San Martin (a tropical State of Peru) is 64.6\% [1]. Cardiac complications such as chest pain, arrhythmias, pulmonary edema and refractory shock have been reported in patients with severe disease. However, the frequency and extent of cardiac involvement in leptospirosis, are under-reported and poorly understood, the majority of the cases are asymptomatic. There are no national reports about these topic.

\section{Case Presentation}

A 39-year-old Peruvian marine, who came from and endemic area for leptospirosis and Malaria, a triangular area called VRAEM (Valle del Rio Apurimac, Ene, Mantaro), a tropical region in Peru. His activity was risky in the jungle, he had to drink and bathe with river water, sometimes stagnant, and sleep in open places, putting himself in contact with wild animals, specifically rodents that came to eat the remains of food. Initially he presented to the Navy Hospital with high grade fever, cephalea, and myalgias so he was diagnosed of Malaria Vivax infection non complicated (received Cloroquine and Primaquine), and he was tested for Lepstospirosis, confirmed by positive leptospira IgM, negative IgG and strongly positive Microscopic Agglutination Test, that's why we indicated doxycycline (a 6 days course). On admission we ruled out any hepatic, kidney, pulmonary and cardiac complication, at that point he was presenting a non-complicated Malaria and leptospirosis co- infection. He was readmitted with severe edema of lower limb, acute headache, polymyalgia, and foamy urine.

On examination: Patient in poor general condition, bipalpebral/lower limb edema and bilateral conjunctival injection.

Thorax and Lungs: Normal vesicular murmur for both hemithorax, not rales; Cardiovascular: Rhythmic heart

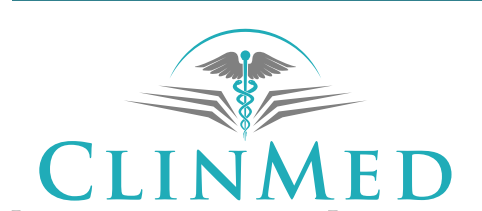

INTERNATIONAL LIBRARY
Citation: Cavalcanti S, L Lerena V, Gomez C (2018) Acute Myopericarditis an Uncommon Presentation of Severe Leptospirosis - A Case Report and Literature Review. Int J Trop Dis 1:009.

Accepted: October 25, 2018; Published: October 27, 2018

Copyright: (C) 2018 Cavalcanti S, et al. This is an open-access article distributed under the terms of the Creative Commons Attribution License, which permits unrestricted use, distribution, and reproduction in any medium, provided the original author and source are credited. 
sounds, tachycardia, no murmurs; Locomotor apparatus: Decrease in strength of lower limbs.

Laboratory: Auxiliary tests: Elisa Ig M leptospirosis: Microagglutination reagent in tube (MAT); Leptospirosis: Autummalis $1 / 400 / C a n i c o l a 1 / 200$; Thoracic radiography: Increased cardiac silhouette with effacement of atrium and right ventricle edges.

During hospitalization, he presents chest pain and dyspnea, echocardiography: pericardial fluid (aprox 300 cc) with no tamponade physiology, thickening of visceral and parietal $(+/-6 \mathrm{~mm})$ pericardium and electrocardiogram: simple and aberrant supraventricular arrhythmia with isolated extrasystoles and elevated cardiac enzymes (Creatine phosphokinase: $600 \mathrm{U} / \mathrm{L}$, Creatine phosphokinase-M: $7 \mathrm{ng} / \mathrm{ml}$, Myoglobin: $120 \mathrm{ng} / \mathrm{ml}$ and Troponin I: $1 \mathrm{ng} / \mathrm{ml}$ ) Proteinuria: $0.62 \mathrm{~g} / 24 \mathrm{~h}$.

All these signs and symptoms associated to the laboratory and image results, and also the Reactive MAT, made us think that we had in front of us a rare manifestation of Severe Leptospirosis, a Myopericarditis due to leptospira. Other possible infective and autoimmune causes were excluded, such as dengue hemorrhagic fever or other arbovirus, autoimmune multi-system disorders such as systemic lupus erythematosus and vasculitis were also differentiated. Patient recovered completely with supportive care, antibiotics and anti-inflammatory medication to ensure the pericardial complication.

\section{Discussion}

Leptospirosis can be considered a generalized, systemic disease, mainly translated by an infectious vasculitis [2]. Vascular lesion, predominantly capillary, is a prominent factor of leptospirosis and responsible for edema and hemorrhagic diathesis. On the other hand, the results of clinical investigations suggest that the severity of leptospirosis could be related to the intensity of the immune response [3]. Highly sensitive and specific MAT is the gold standard serological test for the diagnosis which became strongly positive repeatedly in this patient.

Multiple factors may contribute to clinical manifestations that suggest cardiac involvement, causing diagnostic confusion. A variety of electrocardiographic changes occur in leptospirosis, with atrial fibrillation, atrioventricular conduction blocks and non-specific ventricular repolarization abnormalities being the most common. Echocardiographic evidence of myocardial dysfunction has not been adequately demonstrated. The diagnostic value of cardiac biomarkers is unknown [4].

No specific therapies are available to prevent or treat cardiac involvement in leptospirosis; current management is based on correction of deranged homeostasis and supportive therapy. Specifically in this case, Infectious diseases division co worked with cardiology service, all physicians were agreed to give anti-inflammatory therapy. The treatment for pericarditis has been now well defined with first-line agents represented by nonsteroidal anti-inflammatory drugs plus colchicine, low-dose corticosteroids with slow tapering as second-line agents and for specific indications [5].

$\mathrm{L}$ interrogans is divided into more than 210 serovars and 23 serogroups. This classification has epidemiological importance since the clinical picture and virulence in general are not related to serovar until now. Recent genetic studies have shown that the taxonomy of the genus Leptospira is more complex, having been able to differentiate 8 pathogenic and 5 non-pathogenic species [6]. It is not described in literature about the association of severe manifestations and specific leptospira serovar involved, or when there is more than one infectious serovar in the same patient (as in the case of our patient who presented positive MAT reaction for autummalis and canicola); much less described is the association of cardiac events with a specific serovar of leptospira.

In this case, although at the first admission the patient did not present any cardiac complication or another organ, proven in the clinical examination and the auxiliary exams, that, does not exclude the fact that days later the picture got complicated. The disease caused by Leptospira Interrogans has two clinical stages, and it is specifically in the second week, what is called the immune phase, when sometimes patients get worse. It is very important to maintain surveillance for at least 4 weeks after the febrile stage in patients with a probable or confirmed diagnosis of Leptospirosis, because they can decompensate after the febrile stage and even after receiving specific antibiotics; also, these two stages get confused and many times become one, when patients are predisposed to get complicated.

A search was made in Pub Med, with the variables myopericarditis and leptospirosis, and just two case report was found, from Constans and Lucht called [Myopericarditis and atrial fibrillation disclosing leptospirosis septicemia] publicated in 1996 - France [7,8], it is curious that both papers had the same title and were publicated in the same year. A croatic study in 2011 that include 97 patients and studied Electrocardiographic changes in hospitalized patients with leptospirosis over a 10-year period, found just 4 of 97 patients with myopericarditis, but they didn't describe them specifically [9]. Thus, this case is the first ever report of leptospirosis presenting acute myocarditis and pericarditis due to Leptospirosis in Peru, and in the last 20 years around the world.

\section{Conclusion}

In conclusion we must consider leptospirosis as a cause of myopericarditis in endemic places and if the epidemiological risk is important. May be several cases of "ideopatic myocarditis" are caused because of Leptospira and other parasitic non chagasic infections.

If the patient presents the diagnosis of probable leptospirosis, we should always consider discarding cardiac 
involvement, taking an EKG and cardiac enzymes, with much more interest if the patient is becoming complicated. Evidence suggests that direct myocardial damage occurs in patients with severe leptospirosis, and further studies are recommended to elucidate its pathophysiology, clinical features and contribution to overall prognosis, and to identify appropriate diagnostic investigations and specific therapies.

\section{References}

1. Jorge O Alarcón-Villaverde, Franco Romani-Romani, Romina A Tejada, Paolo Wong-Chero, Manuel Céspedes-Zambrano (2014) Seroprevalencia de leptospirosis y características asociadas en agricultores de arroz de una región tropical del Peru. Rev Med Exp Saludpublica 31: 195-203.

2. Andre V Lomar, Decio Diament, Jaime R Torres (2000) Leptospirosis in Latin America. Emerging and re-emerging Diseases in Latin America. Infect Dis Clin 14: 23-39.

3. Abdulkader RC, Daher EF, Camargo ED, Spinosa C, da
Silva MV (2002) Leptospirosis severity may be associated with the intensity of humoral immune response. Rev Inst Med Trop Sao Paulo 44: 79-83.

4. Shah K, Amonkar GP, Kamat RN, Deshpande JR (2010) Cardiac findings in leptospirosis. J ClinPathol 63: 119-123.

5. Galluzzo A, Imazio M (2018) Advances in medical therapy for pericardial diseases. Expert Rev Cardiovasc Ther 16: 635-643.

6. Adelina Braselli. Leptospirosis.

7. Lucht F, Lafond P, Bertrand JC (1996) Myopericarditis and auricular fibrillation disclosing septicemic leptospirosis. Presse Med 25: 1805.

8. Constans J, Gosse P, Cottarre M, Ansoborlo P, Mercie P, et al. (1996) Myopericarditis and atrial fibrillation disclosing leptospirosis septicemia. Presse Med 25: 555.

9. Škerk V, Markotić A, Puljiz I, Kuzman I, Čeljuska Tošev E, et al. (2011) Electrocardiographic changes in hospitalized patients with leptospirosis over a 10-year period. Med Sci Monit 17: 369-375. 